\title{
Stop the Sores: The Making and Evaluation of a Successful Social Marketing Campaign
}

\author{
Aaron Plant, MPH \\ Jorge A. Montoya, $\mathrm{PhD}$ \\ Harlan Rotblatt \\ Peter R. Kerndt, MD, MPH \\ Karen L. Mall, MBA \\ Les G. Pappas, MPA \\ Charlotte K. Kent, PhD \\ Jeffrey D. Klausner, MD, MPH
}

Los Angeles County has experienced a rapid increase in early syphilis among men who have sex with men (MSM) in recent years, with the number of cases rising from 126 in 2000 to 809 in 2005. As part of the public health response to this outbreak, a sustained social marketing campaign was launched in 2002, with the objectives of increasing syphilis testing, knowledge, and awareness among MSM in Los Angeles. This campaign, as implemented, exemplified key principles of social marketing, including market research, audience segmentation, and branding. A cross-sectional study conducted in 2004 to evaluate the campaign found that those MSM who were aware of the campaign were nearly twice as likely to have tested for syphilis in the past 6 months as those MSM who were not aware of the campaign. Those MSM who were aware of the campaign also had more syphilis awareness and knowledge in key areas.

Keywords: social marketing; men who have sex with men; syphilis; sexually transmitted disease; evaluation; intervention; branding

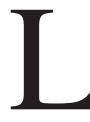

os Angeles County has experienced a rapid increase in early syphilis among men who have sex with men (MSM) in recent years, with the number of cases rising from 126 in 2000 to 809 in 2005 (Sexually

Health Promotion Practice

Month XXXX Vol. XX, No. X, xx-Xx

DOI: $10.1177 / 1524839907309376$

(C)2008 Society for Public Health Education
Transmitted Disease Program [STDP], Los Angeles County Department of Public Health, 2006). In 2005, MSM comprised $66 \%$ of all early syphilis cases in Los Angeles County. This alarming trend mirrors rising syphilis incidence among MSM in major cities across the United States, Europe, and Australia (Centers for Disease Control and Prevention, 2002, 2004; Jin et al., 2005; Simms et al., 2005; van der Bij, Stolte, Coutinho, \& Dukers, 2005). Although syphilis is a serious disease with long-term consequences if untreated, this increase is particularly worrisome because syphilis is known to facilitate the transmission of HIV (Fleming \& Wasserheit, 1999). In Los Angeles, approximately $60 \%$ of MSM diagnosed with early syphilis in 2005 were also HIV positive (STDP, Los Angeles County Department of Public Health, 2006). Furthermore, a recent study suggests that acquisition of syphilis and HIV may be occurring concurrently among MSM (Taylor et al., 2005).

As part of its public health response to this syphilis outbreak, the Los Angeles County Department of Public Health STDP partnered with AIDS Healthcare Foundation (AHF) and five other community-based organizations (CBOs) in 2001 to support a social marketing campaign. This article outlines the development, implementation, and evaluation of Stop the Sores, a social marketing campaign designed to increase syphilis testing, knowledge, and awareness among MSM in Los Angeles County. Special attention will be given to the role of social marketing principles in the success of the campaign. The evaluation was conducted to assess the impact of the campaign, if any, on syphilis testing behavior among MSM. Specifically, MSM who were exposed to the campaign should be more likely to have had a syphilis test. 


\section{The Authors}

Aaron Plant, MPH, is a research analyst in communications and program evaluation at the Sexually Transmitted Disease Program, Los Angeles County Department of Public Health in Los Angeles, California..

Jorge A. Montoya, PhD, is director of communications and program evaluation at the Sexually Transmitted Disease Program, Los Angeles County Department of Public Health and research assistant professor at the University of Southern California in the Department of Psychology in Los Angeles, California.

Harlan Rotblatt is a project director for communications and program evaluation at the Sexually Transmitted Disease Program, Los Angeles County Department of Public Health in Los Angeles, California.

Peter R. Kerndt, MD, MPH, is the director of the Sexually Transmitted Disease Program in the Los Angeles County Department of Public Health, associate clinical professor in the Department of Medicine, Division of Infectious Disease at the University of Southern California, and adjunct associate professor in the University of California, Los Angeles School of Public Health, Department of Epidemiology in Los Angeles, California.

Karen L. Mall, MBA, is the director of the Prevention \& Testing Department for AIDS Healthcare Foundation, Los Angeles, California.

Les G. Pappas, MPA, is the president and creative director at Better World Advertising, a social marketing firm based in San Francisco, California.

Charlotte K. Kent, PhD, is the chief of the Epidemiology Unit, STD Services, San Francisco Department of Public Health in San Francisco, California.

Jeffrey D. Klausner, MD, MPH, is the director of STD Services, San Francisco Department of Public Health and an associate clinical professor of medicine, Divisions of Infectious Diseases \& AIDS, University of California, San Francisco, California.

\section{BACKGROUND}

\section{Elements of Successful Social Marketing Campaigns}

Social marketing is a consumer-centered, researchdriven approach to promote voluntary behavior change in a priority population (Grier \& Bryant, 2005; Lamptey \& Price, 1998). Although marketing in the private sector is primarily concerned with compelling consumers to purchase a product that has an immediate, tangible reward, social marketing is challenged with changing consumer behavior for benefits that are not necessarily guaranteed and may not become apparent for years. Still, social marketing has its foundation in consumer marketing and the two approaches share many basic components.

Successful social marketing campaigns employ a "marketing mix," adapted from commercial marketing, that includes the product, price, place, and promotion (Grier \& Bryant, 2005). In addition, other key elements of social marketing include market research (Walsh, Rudd, Moeykens, \& Moloney, 1993), audience segmentation (Grier \& Bryant, 2005), and branding (Keller, 1998). The most fundamental characteristic of social marketing is rigorous market research (Walsh et al., 1993). This research is necessary in the formative stage of a campaign so that the values, attitudes, and beliefs of the priority audience can be understood and barriers to adopting the desired behavior (product) can be discovered. Research does not end at the formative stage, however. Feedback from the priority audience must be continually gathered throughout the life of the campaign, and the campaign should be regularly refined in response to new information (Walsh et al., 1993). Another important element of social marketing is segmentation of the intended audience based on variations in what members need, want, and value (Grier \& Bryant, 2005). Segmentation is essential for developing campaign messages that resonate with the priority population and that, for example, are tailored to their current stage of behavior change. Customizing messages in this manner helps ensure that the product is appealing and applicable to each subgroup in the priority population. Moreover, proper segmentation can identify the largest or highest-risk subgroup so that limited resources can be optimally used.

Considering its importance to commercial marketing, the concept of branding has been given insufficient attention in social marketing literature. Much more than simply a name or logo, a brand comprises the range of emotional responses evoked by a product (McDivitt, 2003). A strong brand can help a social marketing campaign stand out and can greatly expand its reach and impact (Evans, Price, \& Blahut, 2005). For instance, a brand has the power to unleash a "set of associations in consumers" minds" (Keller, 1998). In this way, a brand becomes a kind of mental shorthand, conveying messages, meaning, and emotions about a product in a succinct, easy-to-recall manner. With regard to social marketing, branding can help make the "benefits of socially desirable behavior more immediate and more tangible to an individual" (Keller, 1998). Thus, a strong brand can help overcome one of the major obstacles of social marketing, making the benefits of a health behavior salient enough for the priority audience to adopt it. The most effective brands will appeal to consumers on an emotional level, providing an 
even stronger incentive to adopt a behavior (Aaker, 1996). Campaigns that strategically converge product, price, place, and promotion with the requisite market research, audience segmentation, and branding produce a marketing mix that is most likely to be successful.

Although social marketing literature in general is quite voluminous, the social marketing literature relating to MSM and syphilis is quite limited. A 1998 article by Lamptey and Price, although not addressing syphilis specifically, offers a good overview regarding the application of social marketing principles to STD campaigns. More recently, in a review of syphilis social marketing efforts in eight U.S. cities, Vega and Roland (2005) report that $71 \%$ to $80 \%$ of MSM were aware of the campaigns, with $45 \%$ to $53 \%$ reporting syphilis testing because of the campaigns. Because the authors do not explain the methodology employed by each jurisdiction, however, these results should be viewed with caution. Finally, two evaluation studies of San Francisco's Healthy Penis campaign (Ahrens et al., 2006; Montoya et al., 2005), the sister campaign to Stop the Sores, both found an association between campaign awareness and syphilis testing.

\section{Campaign Development and Implementation}

In 2001, AHF and five other CBOs serving MSM in Los Angeles County joined with the STDP to develop a social marketing campaign to quell the rising rates of syphilis among MSM. Beginning as an informal working group, $\mathrm{AHF}$ and the other five community organizations formed the Syphilis Media Organizing Committee (SMOC) in 2002, which served as a community advisory board for the new social marketing campaign. The participation of these six community organizations, which collectively were representative of and had extensive experience serving Los Angeles County's ethnically diverse MSM population, offered credibility to the campaign. The SMOC also lent considerable expertise with developing campaign objectives and messages, assessing the appropriateness of campaign materials to the different segments of the priority population, and distribution of campaign materials. Based on input from SMOC members, the campaign was developed to be sex positive, educational regarding syphilis, and focused more on increasing syphilis testing than on changing sexual behavior to prevent syphilis transmission. SMOC members argued that local media was already saturated with safer sex messages from HIV prevention campaigns for MSM, and felt that a secondary prevention strategy would have the most direct impact on reducing the spread of syphilis. Because of this contention, SMOC members and the STDP determined that the primary campaign product message should be "get tested for syphilis," with the objective of increasing syphilis testing among MSM in Los Angeles County. Secondary objectives were to increase awareness about the growing syphilis epidemic in gay and bisexual men, and to increase knowledge about syphilis (prevention, transmission, symptoms, and complications).

Better World Advertising (BWA), a firm specializing in social marketing, was retained to develop campaign concepts. Stop the Sores was drawn from a closely related antisyphilis campaign concept, Healthy Penis, that was developed by BWA for consideration by the STDP and SMOC members, and by the San Francisco Department of Public Health. The Healthy Penis concept had been well received by two focus groups of MSM. Stop the Sores shared common objectives, messages, and visuals with Healthy Penis and featured a personified syphilis chancre cartoon character from the Healthy Penis campaign named Phil the Sore. Stop the Sores was perceived by SMOC members and the STDP as having several key benefits, including (a) the use of humor to counter HIV prevention burn out among MSM and as a positive alternative to fear-based, negative, or heavily sexualized HIV prevention ads for MSM; (b) sustainability over at least a year, through use of a serial cartoon narrative; (c) flexibility to include a range of information about syphilis while stressing the main message about syphilis testing; and (d) ability to create a strong brand by promoting the campaign in various media channels, along with use of a life-sized Phil costumed character for regular campaign outreach. The Stop the Sores campaign was launched in June 2002 to coincide with the gay pride festival in West Hollywood. Healthy Penis was concurrently launched to combat rising syphilis rates in San Francisco (Montoya et al., 2005).

The Stop the Sores campaign was intended to appeal to the wide range of ethnic groups of MSM in Los Angeles. The initial campaign materials were tested in two ethnically diverse focus groups in 2002 and were also approved by a diverse panel of SMOC members. To reach the large segment of Spanish-speaking MSM, all campaign ads and the entire Stop the Sores Web site were translated into Spanish by BWA, then reviewed for cultural appropriateness by Spanish-speaking MSM in the SMOC and STDP.

BWA incorporated information from the two initial focus groups and input from the STDP and SMOC in developing the Stop the Sores brand. One consideration in branding a social marketing campaign is whether to brand the desirable behavior, the undesirable behavior, or both (Keller, 1998). BWA branded the positive behavior of syphilis testing, and the negative consequences of not testing, exemplified by Phil the Sore. The campaign materials depicted gay characters in various realistic situations, such as going on a date, to the gym, or to a party, 
to encourage the priority audience to identify personally with the campaign. While the men depicted in the campaign went about their daily routines, however, Phil the Sore was always lurking somewhere nearby. Phil was characterized as very sneaky, conniving, and dangerous; he and his legion of sores were constantly searching for unsuspecting gay men to infect with syphilis. To heighten MSM's concern about syphilis infection, Phil was portrayed as a close partner of HIV and as leading to brain and heart damage if left untreated. Although Phil was repulsive, Stop the Sores used humor to keep the overall campaign attractive. A key element of the campaign's brand strategy was to employ a humorous tone to break through "HIV prevention fatigue" and set Stop the Sores apart from other serious health messages aimed at MSM that often use fear appeals. The use of humor in health messages helps to gain the attention of the priority audience, creates receptivity to campaign messages, and may even help to facilitate behavior change (Monahan, 1995).

According to Keller (1998), branding in social marketing campaigns "will have the most impact when individuals seem to be overlooking positive considerations and downplaying negative considerations with respect to the public health issue." In addition to highlighting the problems syphilis can pose for MSM, Stop the Sores offered its product, syphilis testing, as a simple solution. Stop the Sores did not ask MSM to change their current sexual behaviors, but rather to adopt a new, protective behavior. The Stop the Sores brand sold syphilis testing by appealing to the emotional attributes of peace of mind and desire for health for gay men and as a way for them to avoid unwittingly infecting their partners. This brand strategy helped to emotionally reinforce the idea that it is in gay men's best interest to be tested for syphilis. To encourage adoption of syphilis testing as a community norm, the campaign depicted a gay character making syphilis testing a regular part of his health routine. The campaign also appealed to gay men's sense of community. Phil had launched an attack on the gay community, and it was up to gay men to "Stop the Sores."

The Stop the Sores campaign was promoted throughout Los Angeles County but with particular emphasis in health districts where the greatest concentration of MSM live and where there are businesses that cater to MSM. These districts are also where most of the syphilis morbidity in MSM is found in Los Angeles County. The campaign was promoted using multiple channels, including advertising in gay publications, posters on streets, in bars, and commercial sex venues, billboards, palm cards, outreach events with a life-sized Phil the Sore costume, a cable television commercial, a Stop the Sores Web site (http://www.stopthesores.org), banner ads on popular gay
Web sites, a telephone hotline, and Phil the Sore squeeze toys. The serial nature of the campaign ads encouraged audience members to follow them each month, with the aim of reinforcing campaign messages and increasing brand awareness. Although the content of the messages in different ads and other media varied from month to month, all materials promoted the product with a "get tested for syphilis" action message. Ads and posters also had information below the cartoon about the transmission and symptoms of syphilis, stated that syphilis is easily cured, and included the Web site address and hotline number (see Figures 1 to 3 ). The easy cure for syphilis message reinforced the low price of the product. In terms of place, the Web site and telephone hotline had additional information including hours and locations for syphilis testing and treatment sites in Los Angeles County. The STDP also funded extended clinic hours for syphilis testing and helped develop new opportunities for syphilis testing through clinics that serve MSM and a mobile testing van. These efforts made syphilis testing more convenient, thereby reducing the price of the product even more.

Stop the Sores was further promoted by using the commercial media to obtain free publicity. The campaign was covered by local and national newspapers and magazines, including the Los Angeles Times, Newsweek, Playboy, and Adweek. Stop the Sores also received radio and television coverage, and the Phil the Sore mascot was featured on The Daily Show, resulting in more highprofile publicity for the campaign. Finally, campaign planners employed media advocacy in November 2004, when several local network television stations refused to air a Stop the Sores television commercial that had already been shown extensively on cable TV. AHF, with STDP support, conducted a press conference that generated a profusion of free exposure for the campaign on a local, national, and even international level, including editorials in both the Los Angeles Times (December 8, 2004; "Self Censorship and Syphilis," 2004) and the New York Times (December 12, 2004; Rich, 2004). Traffic on the Stop the Sores Web site increased by $1300 \%$ in the months following this press coverage.

Adhering to the consumer-focused principles of social marketing, the Stop the Sores campaign evolved with each subsequent year. A second round of focus groups, this time comprised of both ethnically diverse English speaking MSM and Spanish speaking MSM, were held in March 2004 to update the Stop the Sores brand. Campaign materials were made less "cartoonish" and used more realistic images as a result of feedback from these groups (see Figures 2 and 3). Furthermore, the relationship between HIV and syphilis was emphasized more in ads from 2004 onward because this message was 


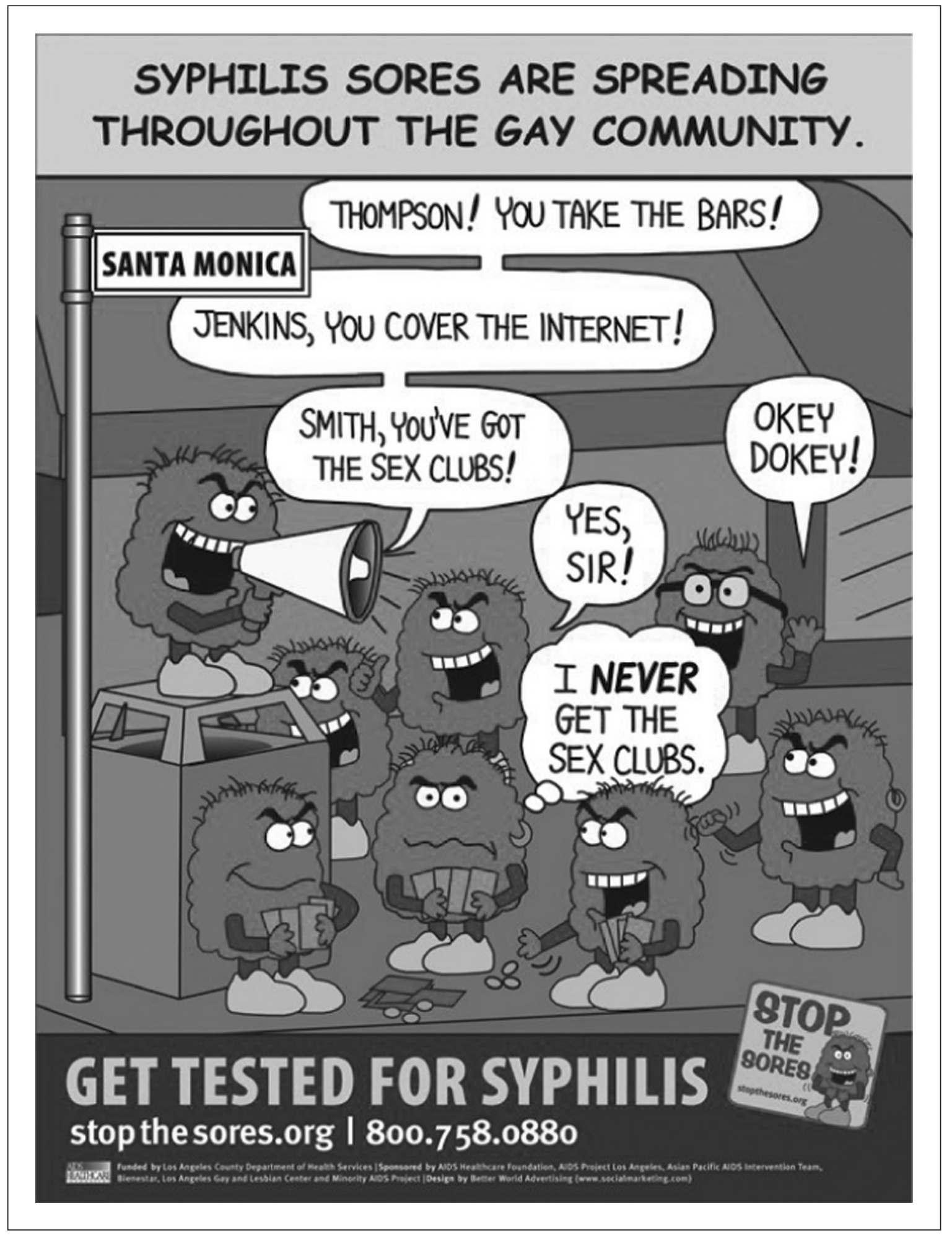

FIGURE 1 Summer 2002 


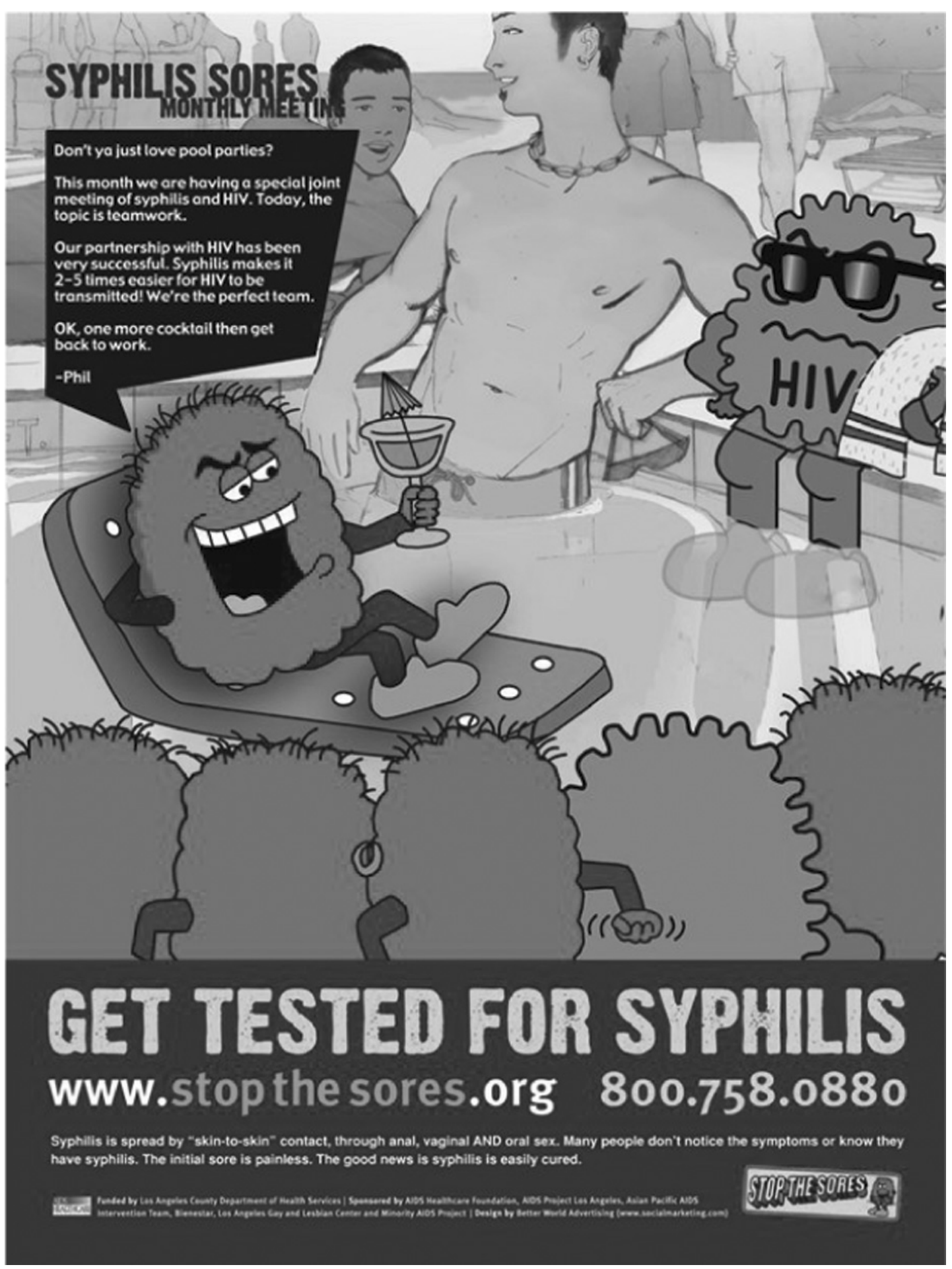

FIGURE 2 Summer 2004 


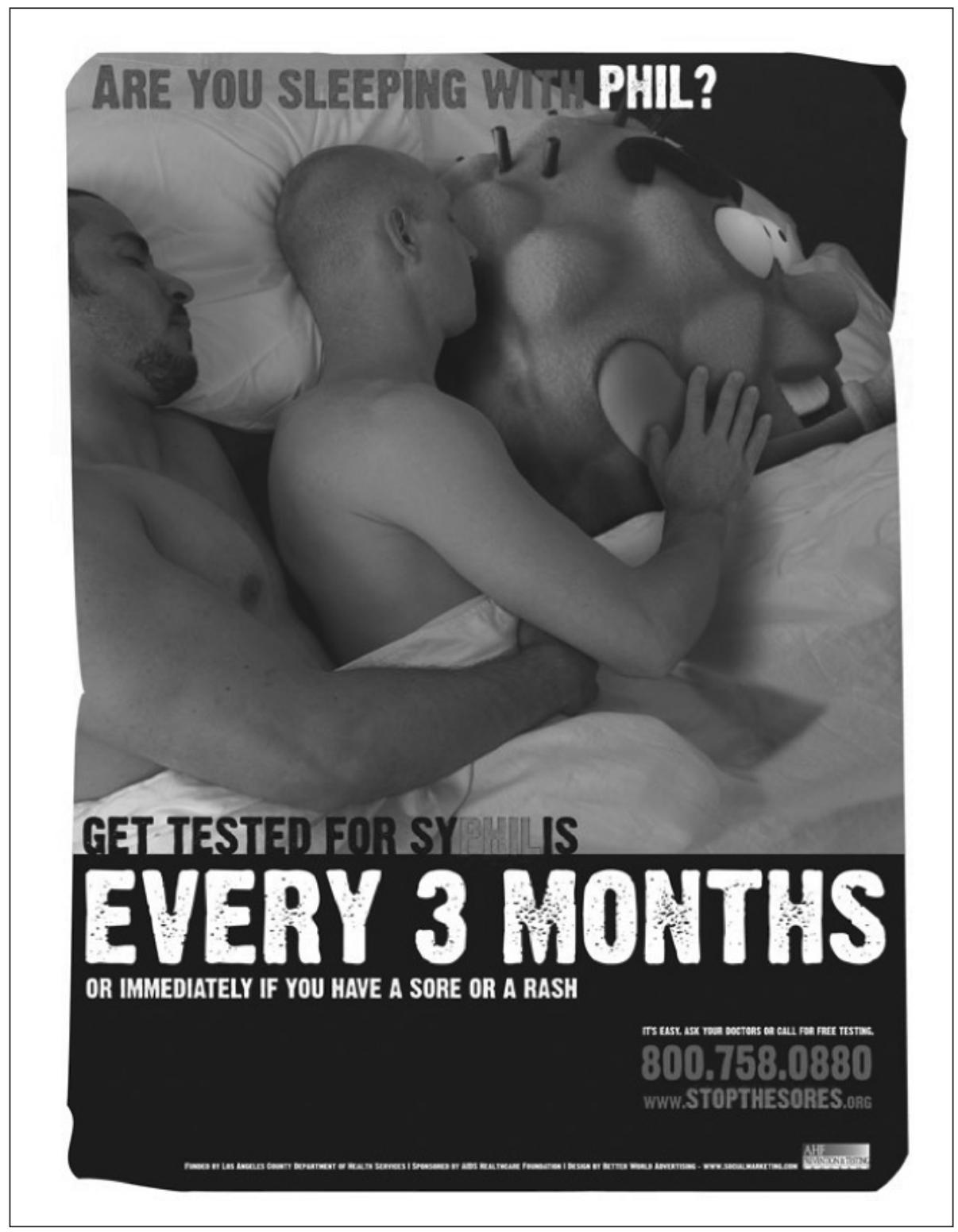

FIGURE 3 Spring 2005

found to be particularly salient by focus group participants (see Figure 2). The changes to campaign materials helped keep Stop the Sores fresh and interesting during its 3-year lifespan.

\section{METHOD}

\section{Sample Recruitment and Eligibility}

The first year of Stop the Sores was evaluated in 2003, with positive syphilis testing behavior, knowledge, and awareness outcomes which were reported elsewhere
(Montoya et al., 2003). To evaluate the effectiveness of the campaign after its second year, a substantially larger convenience sample of respondents was surveyed from October 1, 2004, to December 13, 2004. Men and male-tofemale transgenders were recruited from sidewalks, bars, coffee shops, a community center, bathhouses, sex clubs, and other venues located in neighborhoods where the campaign was promoted. Interviewers approached all men they encountered at the venue and said they were "asking people in this area some questions about sexual health issues to improve health services in [their] community." Respondents were informed that the interview 
was completely anonymous and that no identifying information would be collected. Respondents were eligible if they were between 18 and 65 years of age, if the gender of their sex partners included males or male-to-female transgenders, and if they lived in Los Angeles County. Each interview took approximately 25 minutes to complete. Participants received $\$ 5.00$ as an incentive and interviewers were paid $\$ 5.00$ for each interview completed.

Data were collected as part of routine public health program evaluation. This activity was therefore designated as public health practice and nonresearch. In accordance with the Code of Federal Regulations, Title 45, Part 46, The Public Service Act, human subjects review is not required for public health nonresearch activities.

\section{Survey Instrument}

The survey instrument was developed by the STDP in collaboration with the San Francisco Department of Public Health. The instrument was reviewed by the SMOC, pilot tested with $10 \mathrm{MSM}$, and several minor changes were made. The interview began with several demographic questions, then assessed Stop the Sores campaign awareness. If persons spontaneously mentioned either "Stop the Sores" or "Phil the Sore" verbatim when asked to "recall any ads or public events that provided information about sexual health issues since June of last year here in Los Angeles," they were classified as having unaided awareness. If the campaign was not mentioned, persons were asked if they could "recall any announcements or events called Stop the Sores" and shown a campaign image. Persons responding affirmatively to this question were classified as having aided awareness. Next, open-ended questions were asked about sources of campaign exposure, frequency of exposure, and perceived key messages. Respondents were then asked to rate campaign usefulness and appeal on a 5point scale. Syphilis knowledge, including knowledge of the connection between HIV and syphilis, was assessed through a series of open-ended questions. The interview also included questions about personal sexual practices, respondent HIV status, and how many times respondents were tested for syphilis in the past 6 months.

\section{Survey Analyses}

Frequencies, chi-square tests, and logistic regressions were performed using SPSS 11.0 for Windows to examine the relationship between campaign exposure (awareness) and syphilis testing behavior, knowledge, and awareness. Data analysis was based on the number of respondents for each question; missing values were excluded.

\section{RESULTS}

\section{Survey Population}

A total of 297 interviews were completed and included in the analyses. The sample comprised $91 \%$ males and $9 \%$ male-to-female transgenders. Of the sample, $98 \%$ reported having male partners, $15 \%$ reported female partners, and $4 \%$ reported transgender partners. A plurality of participants were White (37\%), followed by Latino or Hispanic (31\%), African-American (20\%), Asian or Pacific Islander $(6 \%)$, and other race or ethnic background $(5 \%)$. The median age of participants was 32 years, ranging from 18 to 64 years. The majority reported a negative HIV status (73\%), followed by $19 \%$ reporting a positive HIV status, $5 \%$ unsure of their status, and $3 \%$ refusing to provide their status. Of those persons approached by interviewers, $35 \%$ refused to participate.

\section{Campaign Awareness}

A majority (71\%) of the sample was aware of Stop the Sores. In all, $27 \%$ spontaneously mentioned the campaign (unaided awareness), and an additional 44\% remembered the campaign (aided awareness) when prompted with a campaign image. Respondents who were aware of the campaign reported being exposed to it an average of eight times in the past 3 months $(M d n=2)$. The evaluation sample also displayed high levels of other important brand awareness indicators. Stop the Sores had a 20\% "brand dominance" among the total sample of respondents, meaning that it was the only specific sexual health campaign respondents could spontaneously recall (Aaker, 1996). Stop the Sores was also "top of mind" for $16 \%$ of respondents, meaning that it was the first campaign a particular participant listed (Aaker, 1996). Respondents mentioned four other specific sexual health campaigns by name, however, the next most frequently mentioned campaign had just $6 \%$ brand dominance and 3\% top of mind awareness. Finally, Stop the Sores campaign awareness rose significantly from the first evaluation, which found a total awareness level of $61 \%\left(\chi^{2}=4.34, p=.037\right)$.

\section{Campaign Usefulness and Appeal}

When asked to rate the usefulness of the campaign on a 5-point scale from not at all useful to very useful, $50 \%$ of respondents who were aware of Stop the Sores said it was very useful and an additional $33 \%$ said the campaign was somewhat useful. When asked to rate the appeal of the campaign on a 5-point scale from not at all appealing to very appealing, $41 \%$ of respondents said the campaign was very appealing, and $33 \%$ said it was somewhat appealing. 
TABLE 1

Predictors of Syphilis Test in the Past 6 Months: Multivariate Analysis

\begin{tabular}{|c|c|c|c|c|}
\hline \multirow[b]{2}{*}{ Variable } & \multirow[b]{2}{*}{ Odds Ratio } & \multicolumn{2}{|c|}{$95 \%$ Confidence Interval } & \multirow[b]{2}{*}{ p Value } \\
\hline & & Lower & Upper & \\
\hline Campaign awareness ${ }^{\mathrm{a}}$ & 1.83 & 1.05 & 3.20 & .033 \\
\hline \multicolumn{5}{|l|}{ Race/ethnicity } \\
\hline White & Reference & - & - & - \\
\hline Asian or Pacific Islander & 0.70 & 0.23 & 2.14 & .528 \\
\hline African American & 1.03 & 0.51 & 2.07 & .940 \\
\hline Latino or Hispanic & 0.93 & 0.51 & 1.71 & .819 \\
\hline Age & 1.00 & 0.97 & 1.03 & .887 \\
\hline \multicolumn{5}{|l|}{ HIV status } \\
\hline Negative & Reference & - & - & - \\
\hline Positive & 1.63 & 0.83 & 3.19 & .156 \\
\hline Unsure & 0.07 & 0.01 & 0.61 & .016 \\
\hline Refused & 0.66 & 0.14 & 3.20 & .607 \\
\hline \multicolumn{5}{|l|}{ Commercial sex } \\
\hline venue visits ${ }^{\mathrm{b}}$ & 1.02 & 0.89 & 1.17 & .807 \\
\hline Internet partners & 1.14 & 0.91 & 1.43 & .259 \\
\hline
\end{tabular}

NOTE: $n=277$.

a. Includes both aided and unaided awareness.

b. Commercial sex venue visits was included because of testing availability at these venues.

\section{Syphilis Knowledge}

Respondents who were aware of the campaign demonstrated significantly more knowledge and awareness than those unaware of the campaign in several areas. Those aware of the campaign were significantly more likely to mention contact with sores $\left(\chi^{2}=7.048, p=.008\right)$ and anal $\operatorname{sex}\left(\chi^{2}=8.217, p=.004\right)$ as modes of transmission for syphilis, damage to the brain $\left(\chi^{2}=5.404, p=.020\right)$, and nervous system $\left(\chi^{2}=7.115, p=.008\right)$ as effects of untreated syphilis, and that the recent rise in syphilis cases in Los Angeles occurred among MSM $\left(\chi^{2}=5.671, p=.017\right)$. In contrast, those not aware of the campaign were significantly more likely to incorrectly believe that the recent rise in syphilis cases had occurred in women $\left(\chi^{2}=6.533\right.$, $p=.011)$. Furthermore, respondents who were aware of the campaign knew both that syphilis makes it easier to "get" $\left(\chi^{2}=13.406, p<.000\right)$ and "spread" HIV $\left(\chi^{2}=4.526\right.$, $p=.033$ ). Because the HIV-syphilis connection was heavily emphasized in the second year of the campaign, a multivariate logistic regression model was used to further investigate the role of campaign awareness in participants' knowledge of the relationship. Controlling for potential confounders such as age, race/ethnicity, HIV status, commercial sex venue attendance, and number of
Internet partners, those aware of the campaign were more than twice as likely (odds ratio $=2.50, p=.004$ ) to know about the connection between syphilis and HIV compared with those not aware of the campaign. No variable in the model other than campaign awareness was found to be significantly associated with knowledge of this connection. Respondents aware of the campaign did not, however, report significantly higher knowledge of early symptoms of syphilis, appropriate risk reduction strategies, or the fact that syphilis is a curable disease.

\section{Syphilis Testing in the Past 6 Months}

Awareness of the campaign was significantly associated with having had a syphilis test in the past 6 months. Of those aware of the campaign, $55 \%$ were tested for syphilis in the past 6 months compared to $39 \%$ of those unaware of the campaign $\left(\chi^{2}=6.53, p=\right.$ .011). After controlling for potential confounders in a multivariate logistic regression model, those aware of the campaign were 1.8 times more likely to have tested for syphilis than those unaware of the campaign (see Table 1). No variable in the model other than campaign awareness was found to be significantly associated with being tested for syphilis. 


\section{$>$ DISCUSSION}

The Stop the Sores campaign was significantly associated with syphilis testing in the past 6 months and with syphilis awareness and knowledge among MSM in Los Angeles County. This effectiveness was likely a result of faithfulness to the principles of social marketing. Care was taken in the campaign to delineate the product, price, place, and promotion, with an emphasis on market research, audience segmentation, and building a strong brand. Stop the Sores was carefully planned with market research used in the formative phase and throughout the process to refine the campaign. The priority audience was segmented by sexual orientation, neighborhood, and into both English and Spanish speakers.

The evaluation also demonstrated that the campaign was effective at creating a strong brand. Awareness is a measure of the strength of a brand's presence in a consumer's mind (Aaker, 1996). A majority (71\%) of gay and bisexual men surveyed was aware of the Stop the Sores campaign and had been repeatedly exposed to it in the past 3 months. This awareness was significantly higher than the awareness level from the previous evaluation (Montoya et al., 2003). More importantly, more than one fourth of the sample spontaneously mentioned the campaign (unaided awareness) when asked to recall any recent sexual health advertisements or events. In addition to having high levels of unaided awareness, respondents demonstrated much higher levels of brand dominance and top of mind awareness for Stop the Sores than for other sexual health campaigns. These indicators show that Stop the Sores was effective in standing out from the glut of sexual health campaigns aimed at MSM in Los Angeles. Analogously, when consumers recall a particular brand (e.g., Coke) when asked to consider a product class (e.g., soft drinks), that brand is much more likely to get on the shopping list (Aaker, 1996). In the same vein, MSM aware of the campaign are likely to spontaneously consider Stop the Sores messages when thinking about their sexual health. Awareness alone, however, does not fully capture the effectiveness of branding. Members of the priority audience must also "link the brand to strong, favorable, and unique associations" (Keller, 1998). In our evaluation, Stop the Sores was found to be useful and appealing by an overwhelming majority of evaluation participants. When respondents were asked to state what they liked most about the Stop the Sores campaign, a common response was that it used humor to discuss a weighty topic. One respondent commented, "They took a serious issue and made it fun to remember."
The evaluation results strongly suggest that Stop the Sores was effective at reaching our primary goal of increasing syphilis testing. Campaign awareness was significantly related to syphilis testing after controlling for potential confounders in a multivariate logistic regression. Specifically, respondents who were aware of the campaign were nearly twice as likely to have been tested for syphilis in the past 6 months as respondents who were unaware of the campaign. This association was sustained over 2 years (Montoya et al., 2003).

These results also suggest the campaign achieved its secondary goals of increasing knowledge and awareness of syphilis among gay and bisexual men in several important areas. Respondents who were aware of the campaign demonstrated more knowledge about syphilis in terms of modes of transmission, effects of untreated syphilis, groups most affected by the disease, and the facilitating relationship between syphilis and HIV. For instance, respondents aware of the campaign were able to correctly identify that the recent increase in syphilis in Los Angeles occurred in gay and bisexual men, whereas those unaware of the campaign were significantly more likely to incorrectly identify women as the group with the recent increase. Knowledge gains concerning the effects of untreated syphilis and the relationship between syphilis and HIV are especially important because focus group participants reported that such knowledge would present a strong motivation to test for syphilis. The interviewing technique employed by this evaluation adds validity to our findings. The data were gathered via faceto-face interviews and the interviewer recorded answers without providing participants with a list of possible responses. This method of collecting data reduces the possibility that respondents made "best guess" responses on campaign awareness and syphilis knowledge items.

The evaluation study of the Stop the Sores campaign had some limitations. Although we attempted to recruit a diverse sample of MSM in Los Angeles County, our street intercept sample may not be representative of the population of MSM in Los Angeles at risk for syphilis infection, and therefore our results may not be fully generalized. A convenience sample was chosen at the time because budget limitations precluded a probability sample of MSM. Future evaluations, however, could employ a more rigorous design, such as time-location random sampling, which would produce a more representative sample of this hard-to-reach population and would also be reproducible (McFarland \& Caceres, 2001). A limitation of the cross-sectional design of the study is that only associational conclusions, and not cause-effect relationships, can be established. Finally, although our interview technique 
likely reduced participants' ability to guess when providing answers, the face-to-face nature of the interview may have increased the likelihood of social desirability bias, especially for questions on sensitive topics like personal sexual behaviors.

\section{CONCLUSION}

Despite some limitations, the results of the second Stop the Sores evaluation suggest that the social marketing campaign was successful in reaching its primary objective of increasing syphilis testing and its secondary objectives of increasing syphilis knowledge and awareness among MSM in Los Angeles County. The evaluation demonstrates that adherence to social marketing principles, including product, price, place, and promotion, in addition to market research, audience segmentation, and branding, can help ensure that campaign objectives are met. Moreover, using unprompted questions to assess campaign awareness and knowledge should become a standard in social marketing evaluation. Future social marketing campaigns would benefit from more rigorous evaluations, especially ones that employ probability samples so that results can be generalized.

Clearly, additional effort will be required to reduce the incidence of syphilis in Los Angeles County and other metropolitan areas around the country. Toward this end, Stop the Sores has now been replicated in three other major cities across the United States (http://www .stopthesores.org). This cross-jurisdictional approach should increase brand awareness overall and will hopefully result in fewer infections introduced by individuals who travel between cities.

\section{REFERENCES}

Aaker, D. A. (1996). Building strong brands. New York: Free Press. Ahrens, K., Kent, C. K., Montoya, J. A., Rotblatt, H., McCright, J., Kerndt, P., et al. (2006). Healthy Penis: San Francisco's social marketing campaign to increase syphilis testing among gay and bisexual men. PLoS Medicine, 3(12), e474.

Centers for Disease Control and Prevention. (2002, September 27). Primary and secondary syphilis among men who have sex with men-New York City, 2001. Morbidity and Mortality Weekly Report, 51(38), 853-856.

Centers for Disease Control and Prevention. (2004, July 9). Trends in primary and secondary syphilis and HIV infections in men who have sex with men-San Francisco and Los Angeles, California, 1998-2002. Morbidity and Mortality Weekly Report, 53(26), 575-578.

Evans, W. D., Price, S., \& Blahut, S. (2005). Evaluating the Truth brand. Journal of Health Communication, 10, 181-192.

Fleming, D. T., \& Wasserheit, J. N. (1999). From epidemiological synergy to public health policy and practice: The contribution of other sexually transmitted diseases to sexual transmission of HIV infection. Sexually Transmitted Infections, 75(1), 3-17.
Grier, S., \& Bryant, C. A. (2005). Social marketing in public health. Annual Review of Public Health, 26, 319-339.

Jin, F., Prestage, G. P., Kippax, S. C., Pell, C. M., Donovan, B. J., Kaldor, J. M., et al. (2005). Epidemic syphilis among homosexually active men in Sydney. Medical Journal of Australia, 183(4), 179-183.

Keller, K. L. (1998). Branding perspectives on social marketing. Advances in Consumer Research, 25, 299-302.

Lamptey, P. R., \& Price, J. E. (1998). Social marketing sexually transmitted disease and HIV prevention: A consumer-centered approach to achieving behaviour change. AIDS, 12(Suppl. 2), S1-S9.

McDivitt, J. (2003). Is there a role for branding in social marketing? Social Marketing Quarterly, 9(3), 11-17.

McFarland, W., \& Caceres, C. F. (2001). HIV surveillance among men who have sex with men. AIDS, 15(Suppl. 3), S23-S32.

Monahan, J. L. (1995). Thinking positively: Using positive affect when designing health messages. In E. Maibach \& R. L. Parrott (Eds.), Designing health messages: Approaches from communication theory and public health practice (pp. 81-98). Thousand Oaks, CA: Sage.

Montoya, J. A., Kent, C. K., Rotblatt, H., McCright, J., Kerndt, P. R., \& Klausner, J. D. (2005). Social marketing campaign significantly associated with increases in syphilis testing among gay and bisexual men in San Francisco. Sexually Transmitted Diseases, 32(7), 395-399.

Montoya, J. A., Rotblatt, H., Kent, C. K., Mall, K., Klausner, J. D., \& Kerndt, P. R. (2003, July). Evaluating "Stop The Sores," a communityled social marketing campaign to prevent syphilis among men who have sex with men, Los Angeles County, 2002-2003. Paper presented at the International Society for Sexually Transmitted Disease Research Congress, Ottawa, Ontario, Canada.

Rich, F. (2004, December 12). The plot against sex in America. New York Times, p. 2.1.

Self censorship and syphilis. (2004, December 8). Los Angeles Times, p. B10.

Sexually Transmitted Disease Program, Los Angeles County Department of Public Health. (2006, April). Early syphilis surveillance summary. Los Angeles: Author.

Simms, I., Fenton, K. A., Ashton, M., Turner, K. M., CrawleyBoevey, E. E., Gorton, R., et al. (2005). The re-emergence of syphilis in the United Kingdom: The new epidemic phases. Sexually Transmitted Diseases, 32(4), 220-226.

Taylor, M. M., Hawkins, K., Gonzalez, A., Buchacz, K., Aynalem, G., Smith, L. V., et al. (2005). Use of the serologic testing algorithm for recent HIV seroconversion (STARHS) to identify recently acquired HIV infections in men with early syphilis in Los Angeles County. Journal of Acquired Immune Deficiency Syndrome, 38(5), 505-508.

van der Bij, A. K., Stolte, I. G., Coutinho, R. A., \& Dukers, N. H. (2005). Increase of sexually transmitted infections, but not HIV, among young homosexual men in Amsterdam: Are STIs still reliable markers for HIV transmission? Sexually Transmitted Infections, 81, 34-37.

Vega, M. Y., \& Roland, E. L. (2005). Social marketing techniques for public health communication: A review of syphilis awareness campaigns in 8 US cities. Sexually Transmitted Diseases, 32(Suppl. 10), S30-S36.

Walsh, D. C., Rudd, R. E., Moeykens, B. A., \& Moloney, T. W. (1993). Social marketing for public health. Health Affairs, 12(2), 104-119. 